\title{
El legado de Luria y la neuropsicología escolar
}

\author{
Dionisio Manga ${ }^{1 *}$ y Francisco Ramos ${ }^{2}$
}

\author{
${ }^{1}$ Universidad de León ${ }^{2}$ Universidad de Salamanca
}

(Recibido 14 Marzo 2011; Aceptado 28 Abril 2011)

RESUMEN: Se revisa la historia de la neuropsicología aplicada al trabajo con los niños. Diversos autores se han mostrado partidarios de aplicar la neuropsicología a los centros educativos, especialidad que algunos han llamado "neuropsicología escolar". Desde que inicialmente así se la denominó (Hynd y Obrzut, 1981), han tenido lugar notables avances respecto al campo e identidad profesional de esta especialidad. La obra de Luria continúa teniendo una gran influencia internacional. Luria ha contribuido a la comprensión neuropsicológica del individuo y de la práctica profesional. Como actividad profesional, la neuropsicología escolar se ocupa de la evaluación, diagnóstico e intervención en estudiantes que tienen dificultades en sus aprendizajes y/o muestran trastornos de comportamiento en su centro escolar. En los últimos años se viene recomendando una evaluación neuropsicológica comprehensiva de cara a la intervención en el centro escolar. Existen procedimientos más nuevos de evaluación neuropsicológica infantil, los cuales representan aplicaciones innovadoras del modelo conceptual de Luria. La investigación y avances de los estudios de neuroimagen nos permiten conocer mejor los cambios del cerebro en desarrollo.

Palabras clave: neuropsicología infantil, neuropsicología escolar, desarrollo del cerebro, modelo conceptual de Luria, evaluación neuropsicológica del desarrollo.

\section{The Luria's legacy and school neuropsychology}

ABSTRACT: This article reviews the history of neuropsychology applied to work with children. Several authors have advocated for the application of neuropsychology to the school centres, a specialty some have called "school neuropsycology". Since the term was initially introduced (Hynd \& Obrzut, 1981), considerable advances have arisen regarding the field and professional identity of this specialty. Luria'work continues to have a strong international influence. Luria has served for neuropsychological understanding of the individual and for the professional practice. As professional activity, the school neuropsychology focuses in assessing, diagnosing, and intervening with students who are experiencing learning difficulties and/or behaviour disorders in academic setting. In recent years, a comprehensive neuropsychological assessment for intervention is recommended within a school setting. Newer neuropsychological assessment procedures for children represent innovative applications of Luria's conceptual model. Research and advances in neuroimaging allow us to view how the developmental brain changes.

Keywords: child neuropsychology, school neuropsychology, brain development, Luria's conceptual model, developmental neuropsychological assessment. 


\section{LA NEUROPSICOLOGÍA ESCOLAR EN SUS COMIENZOS}

Gaddes abogaba al inicio de la década de 1980 por "el uso del conocimiento neuropsicológico para entender los trastornos del aprendizaje" (Gaddes, 1980, p. 75). Se trataba, en definitiva, de incrementar el conocimiento de las relaciones cerebro-conducta en el contexto de los problemas del funcionamiento cognitivo y del aprendizaje escolar. Aunque Gaddes reconocía que el intento de relacionar el conocimiento neurológico, el psicológico y el educativo aún estaba en su infancia, consideraba no obstante que este empeño era de capital importancia para entender mejor y más rápidamente los trastornos del aprendizaje, porque aglutinaba el conocimiento y la experiencia profesional de neurólogos, psicólogos y educadores.

Cuando Hynd y Obrzut (1981) escribieron su artículo titulado Neuropsicología escolar, confesaron que una de sus finalidades era la de aportar un marco conceptual a esta especialidad dentro de la profesión de la psicología escolar. Si entonces el marco conceptual se hallaba poco elaborado, treinta años después podemos referirnos a notables avances en la citada especialidad.

La neuropsicología escolar es una especialidad de la neuropsicología clínica cuando ésta se aplica en los centros educativos, tal como han defendido notables autores desde los comienzos (Hynd y Obrzut, 1981; Gaddes, 1981; Obrzut, 1981). La concepción de Luria sobre la organización del cerebro en desarrollo tuvo gran aceptación ya en la década de 1980. Desde una idea más rehabilitadora, no se tardó en recomendar el modelo de Luria como aplicación neuropsicológica en los servicios de los centros escolares (Obrzut y Obrzut, 1982). Desde una perspectiva más teórica, Hynd y Willis (1988) asumían, en su Neuropsicología pediátrica, que las teorías de organización neuropsicológica en niños derivan de la concepción teórica de Luria.

La primera propuesta teórica es la organización funcional del cerebro, según los bloques funcionales de Luria y su idea de la organización jerárquica en áreas primarias, secundarias y terciarias. Una segunda teoría neuropsicológica aplicable a los niños se refiere, a juicio de Hynd y Willis (1988), a los modos de procesamiento de la información, que partiendo de las síntesis simultáneas y sucesivas de Luria $(1973,1980)$, han abierto una línea de desarrollo seguida principalmente por el grupo de Das (Das y Varnhagen, 1986; Das, Naglieri y Kirby, 1994) en el modelo PASS para evaluación de los procesos cognitivos, así como por Kaufman en el test de inteligencia para niños K-ABC (Kaufman y Kaufman, 1983).

Por fin, Hynd y Willis (1988) apuntan una tercera línea teórica derivada de Luria para aplicación en niños. Se refieren al desarrollo de la especialización hemisférica cerebral. Esta línea ha avanzado enormemente, después de los primeros planteamientos de Luria $(1973,1980)$ sobre la mayor lateralización hemisférica de las áreas secundarias y terciarias. 


\section{EL MODELO DE LURIA Y SU INFLUENCIA EN LA NEUROPSICOLOGÍA INFANTIL}

La concepción neuropsicológica de Luria $(1973,1980)$ asume que el funcionamiento cerebral se lleva a cabo con la participación, conjunta e indispensable, de las tres unidades o bloques funcionales básicos del cerebro. El primero es el bloque de la activación, encargado del tono cortical o estado óptimo de activación de la corteza cerebral. La estructura más importante de este bloque es la formación reticular, tanto ascendente como descendente, sobre todo a causa de sus conexiones con el córtex frontal. La neuropsicología clínica infantil considera que algunos síntomas asociados a trastornos del aprendizaje, tales como la atención evolutivamente inapropiada y la hiperactividad, pueden guardar relación con la disfunción o retraso madurativo de este bloque funcional de la activación.

El segundo bloque funcional, o bloque del input, está al servicio de la recepción, elaboración y almacenamiento de la información. Ocupa las regiones posteriores de la corteza cerebral, concretamente los lóbulos parietal, temporal y occipital, en los que respectivamente se hallan las zonas táctil-cinestésica, auditiva y visual. El tercer bloque, llamado bloque de programación y control de la actividad, abarca los sectores corticales situados por delante de la cisura de Rolando (o central).

En el modelo de Luria las regiones corticales pertenecientes a los bloques segundo y tercero se organizan jerárquicamente en áreas primarias, secundarias y terciarias. En el segundo bloque, a la ley de estructura jerárquica anterior se añade la ley de especificidad decreciente, hasta el punto de que las áreas terciarias no poseen modalidad sensorial específica y son supramodales. La noción de sistemas funcionales resulta igualmente central en la concepción neuropsicológica de Luria (ver, para ampliación, Manga y Ramos, 2000; Semrud-Clikeman y Teeter, 2009).

\section{CAMBIOS EN LA ORGANIZACIÓN FUNCIONAL DEL CEREBRO DU- RANTE EL DESARROLLO}

Golden (1981) ya planteó, siguiendo la organización funcional del cerebro en tres bloques según Luria, cómo discurría dicha organización a lo largo del desarrollo. Más recientemente Semrud-Clikeman y Teeter (2009) hacen suya la propuesta de Golden (1981) sobre cinco etapas del desarrollo en la organización funcional del cerebro. De las dos últimas etapas, en la cuarta, el área parietal terciaria es la responsable de que los niños sean eficientes en la mayoría de las competencias educativas.

La etapa quinta es la del desarrollo de las áreas prefrontales del cerebro. Nivel terciario de la acción intencional y la planificación. Su desarrollo se prolonga hasta la adolescencia. Según Semrud-Clikeman y Teeter (2009), hay autores que sostienen que el comienzo del desarrollo de los lóbulos prefrontales se da ya a los 6 años de edad. "Las etapas neuroevolutivas son de importancia primaria para la 
neuropsicología infantil, y se necesita más investigación para trazar con mayor claridad estas etapas del desarrollo cerebral" (Semrud-Clikeman y Teeter, 2009, p. 165).

Tabla 1. Las Áreas Terciarias Del Cerebro y Sus Funciones (a partir de Luria, 1980; Golden, 1981; Semrud-Clikeman y Teeter, 2009).

\begin{tabular}{|c|c|c|c|}
\hline Bloque & Sistemas Funcionales & $\begin{array}{c}\text { Áreas de } \\
\text { Brodmann }\end{array}$ & $\begin{array}{c}\text { Correlatos } \\
\text { comportamentales }\end{array}$ \\
\hline $\begin{array}{l}\left(2^{\circ}\right) \text { Input. } \\
\text { Recepción, } \\
\text { elaboración y } \\
\text { almacena- } \\
\text { miento de la } \\
\text { información }\end{array}$ & $\begin{array}{l}\text { Región terciaria PTO } \\
\text { (parieto/temporo/occi- } \\
\text { pital) }\end{array}$ & $\begin{array}{l}19,21,22 \\
37,39,40 .\end{array}$ & $\begin{array}{l}\text {-Procesamiento } \\
\text { simultáneo. } \\
\text {-Capacidades, por } \\
\text { ejemplo, de lectura, } \\
\text { escritura, matemáticas, } \\
\text { lenguaje gramatical y } \\
\text { sintáctico, } \\
\text { estereognosia y } \\
\text { rotación espacial. }\end{array}$ \\
\hline $\begin{array}{l}\left(3^{\circ}\right) \text { Output. } \\
\text { Programación } \\
\text { y control de la } \\
\text { actividad }\end{array}$ & $\begin{array}{l}\text { Región terciaria de los } \\
\text { lóbulos frontales } \\
\text { (áreas prefrontales) }\end{array}$ & $\begin{array}{l}9,10,11, \\
12,46,47 .\end{array}$ & $\begin{array}{l}\text {-Toma de decisiones y } \\
\text { valoración } \\
\text {-Control de impulsos } \\
\text {-Retardo de la } \\
\text { gratificación } \\
\text {-Atención focalizada }\end{array}$ \\
\hline
\end{tabular}

\section{EL RECONOCIMIENTO A LA NEUROPSICOLOGÍA DE LURIA A FINALES DEL SIGLO XX}

Cuando el siglo XX llegaba a su fin, aparecieron diferentes artículos en los que no se escatimaban elogios a la utilidad e influencia de las enseñanzas de Luria aplicadas a los niños. Enseñanzas útiles tanto para realizar evaluaciones neuropsicológicas como para recuperar a niños con problemas en sus aprendizajes. Se trata del número especial dedicado a Luria por la revista Neuropsychology Review, en 1999. El trabajo introductorio de Tupper (1999) abordaba la evaluación neuropsicológica después de Luria (nacido éste en 1902 y muerto en 1977 en Moscú). Allí repasaba Tupper las baterías neuropsicológicas que en lengua inglesa han adoptado el modelo de Luria.

En otro trabajo, Glozman (1999) revisaba la integración, junto con sus ventajas para intervenir en escolares, del procedimiento evaluador cuantitativo. Das (1999), a su vez, presentaba en la primera parte de su artículo la batería de tareas para cuatro procesos cognitivos: Planificación, Atención, y procesamiento Simultáneo y Sucesivo (PASS). El modelo PASS se basa en la valoración cualitativa aportada por Luria, en lo que él llamó análisis del síndrome, completándose con tareas obtenidas de la psicología cognitiva y la neuropsicología.

Korkman (1999) mostraba cómo se aplican los principios diagnósticos de Luria a la evaluación neuropsicológica de los niños. De acuerdo con Luria, son 
necesarios ciertos ámbitos de exploración: la atención, las funciones ejecutivas, el lenguaje, las funciones sensorio-motoras, las funciones visoespaciales, la memoria y el aprendizaje. Para tal fin se ha construido una batería de tests neuropsicológicos, denominada NEPSY, cuya última adaptación en lengua inglesa se había realizado el año anterior, para niños de 3 a 12 años (Korkman, Kirk y Kemp, 1998).

En España, Manga y Ramos (1999) publicaron en castellano La evaluación neuropsicológica, en la revista Cínica y Salud. En ese trabajo se describían las baterías que habían adoptado el modelo neuropsicológico de Luria en la evaluación de los niños. La batería Luria-DNI (Diagnóstico Neuropsicológico Infantil siguiendo a Luria), de Manga y Ramos (1991) para niños de 7 a 10 años de edad, se basa en la versión castellana del libro de Christensen (1975), versión revisada por D. Manga y publicada en la editorial Visor (Christensen, 1987) con el título de El Diagnóstico Neuropsicológico de Luria.

Es, por tanto, la publicación de Christensen $(1975,1987)$ la que ha inspirado la construcción de baterías neuropsicológicas para niños: Luria-Nebraska Infantil (Golden, 1981), NEPSY (Korkman et al., 1998), PASS (Das y Varnhagen, 1986; Das et al., 1994; Das, 1999), Luria-DNI (Manga y Ramos, 1991) y LuriaInicial (Manga y Ramos, 2006). Bausela (2008) ha validado la batería LuriaInicial con niños mexicanos.

La batería Luria-DNI, actualmente en proceso de revisión para ser en adelante batería Luria-DNI-R, ha servido a la realización de una docena de tesis doctorales en diferentes universidades españolas, la mayoría leídas y otras en elaboración. Véanse, para su descripción y aplicación: Manga y Ramos (1991) y Manga y Fournier (1997), así como para algunos hallazgos con la misma (Manga y Ramos, 2001; Ramos y Manga, 2003).

\section{MODELOS (INSPIRADOS EN LURIA) ÚTILES PARA LA NEUROPSICO- LOGÍA INFANTIL}

En la actualidad los modelos de la neuropsicología infantil, y por tanto de la escolar, pretenden una consideración del niño dentro de su entorno, con una evaluación amplia que se encamine a la intervención. (Quintanar, Solovieva, Lázaro, Bonilla, Mejía, Eslava, 2008; Witsken, Stoeckel y D’Amato, 2008). La intervención implica trabajar con el escolar y con otras personas significativas en el entorno natural del escolar, enfatizando así la ecología total que le afecta en su rendimiento académico. Esta afirmación nos da una idea clara de la pretensión de la neuropsicología ecológica: "es un acercamiento basado en fortalezas, el cual considera al niño, al igual que a los sistemas con los que interactúa, cuando se evalúa, diagnostica e interviene con estudiantes que pasan por dificultades de aprendizaje" (D’Amato, Crepeau-Hoson, Huang y Geil, 2005, p. 97).

No se ha llegado nunca a un claro acuerdo sobre las áreas específicas que debe abarcar un examen neuropsicológico, amplio y comprehensivo. Mientras algunos se inclinan por evaluar sólo unas pocas áreas críticas, otros, como Luria 
y los continuadores de su modelo usando baterías neuropsicológicas infantiles, son partidarios de evaluar muchos dominios neuropsicológicos, agrupados éstos en las siguientes habilidades o capacidades: (a) funciones motoras, (b) sensopercepción, (c) habilidades de lenguaje y comunicación mediante el habla (d) habilidades con el lenguaje escrito y el cálculo, (e) capacidad de aprendizaje y memoria inmediata, (f) funcionamiento ejecutivo y atención.

La evaluación, a su vez, de las capacidades cognitivas o de inteligencia, permitirán a su vez obtener un perfil cognitivo. A estas capacidades, neuropsicológicas y cognitivas, se ha de añadir, según Witsken et al. (2008), la exploración de otros dos dominios, a saber, personalidad/conducta/familia, más ambiente educativo y de la clase.

Witsken et al. (2008) se apoyan en una reflexión previa de Glozman (1999) cuando afirman que la exploración neuropsicológica de Luria es reconocida actualmente por la comunidad científica internacional como el método más comprehensivo y flexible de la evaluación neuropsicológica disponible, pudiendo abordar los factores que subyacen a las actividades psicológicas complejas. Tranel (2005) afirmaba que, para Luria, el uso de signos revelados por la evaluación neuropsicológica, pudiendo inferir a partir de ellos disfunciones cerebrales, era la verdadera esencia del campo de la neuropsicología.

\section{CAMPOS PREFERENTES DE LA NEUROPSICOLOGÍA ESCOLAR DEL FUTURO}

\section{Las funciones ejecutivas}

Las funciones ejecutivas tienen una naturaleza elusiva, es decir, no definida todavía con la suficiente claridad. Se trata de un concepto que necesita comprenderse con mayor precisión (Jurado y Roselli, 2007). Tales funciones incluyen capacidades de formación de metas, de planificación, de realización de planes y de su ejecución efectiva.

Se reconoce que fue Luria (1973) quien inicialmente asignó funciones ejecutivas a la corteza prefrontal del cerebro. "Los lóbulos frontales del cerebro y, en particular, sus formaciones terciarias (incluyendo el córtex prefrontal) fueron las últimas partes de los hemisferios cerebrales que se formaron... y no alcanzan la madurez en el niño hasta la edad de 4 a 7 años... Los lóbulos frontales tienen la función de formar planes estables e intenciones capaces de controlar el comportamiento consciente posterior del sujeto" (Luria, 1973, pp. 185 y 195 de la versión castellana).

Respecto a las funciones ejecutivas, leemos textualmente en Stuss y Benson: "Luria enfatizó la importancia y diversidad de la función prefrontal, usando diferentes términos como planificación, intención, programación, regulación, y verificación. Luria y otros han hecho casi universalmente aceptable el hecho de que la función de control o función ejecutiva de la conducta humana se halla asociada al córtex prefrontal " (Stuss y Benson, 1990, p. 39). 
Recientemente, Chevalier (2010) deja claro que las funciones ejecutivas se han considerado durante mucho tiempo como una entidad misteriosa, sólo manifestada durante la adolescencia, aunque ahora queda bien establecido que se trata de funciones que implican una multitud de procesos y que las primeras formas de control del pensamiento y de la acción aparecen en etapas muy tempranas del desarrollo. Los estudios que abordan las funciones ejecutivas de los niños las sitúan en estrecha relación con la memoria de trabajo y la atención.

Anderson (2002) ha trazado el perfil evolutivo de los procesos incluidos en la denominada función ejecutiva (FE). La FE incorpora cuatro dominios ejecutivos, separables y muy relacionados entre sí: control atencional, flexibilidad cognitiva, establecimiento de metas y procesamiento de información. Los procesos de cada dominio operan de forma integrada para posibilitar el "control ejecutivo". En los estudios de maduración de los cuatro dominios ejecutivos, se ha encontrado que: (a) el control atencional es el primero en aparecer en etapas tempranas infantiles; (b) los otros tres dominios ejecutivos experimentan un periodo crítico de desarrollo entre los 7 y los 9 años de edad, hasta conseguir un estado de maduración relativa en torno a los 12 años. Todavía se necesitan estudios de neuroimagen para mejor entender la neuropsicología (relaciones cerebro-conducta) del desarrollo de la FE.

\section{Perfiles neuropsicológicos en el TDAH de niños}

El patrón considerado esencial de los déficits cognitivos del TDAH (Trastorno por Déficit de Atención e Hiperactividad), particularmente las deficiencias en tareas de atención y en las funciones ejecutivas, presenta un parecido con el síndrome del lóbulo frontal en los adultos. Esto ha llevado a la hipótesis de que en el TDAH puede darse disfunción cerebral, especialmente en el córtex prefrontal o en las regiones que se proyectan en el córtex prefrontal (Shue y Douglas, 1992).

Recientemente, Goldstein y Naglieri (2008) nos ofrecen la neuropsicología escolar del TDAH. Abordan la teoría sobre el TDAH, así como también su evaluación e intervención neuropsicológicas, mediante los perfiles de los procesos medidos según el modelo PASS.

En niños, las diferencias entre el subtipo combinado (TDAH-C) y el subtipo inatento o con predominio de la inatención (TDAH-I) han interesado en varios trabajos de investigación, algunos en la revista Child Neuropsychology de los últimos años. En uno de ellos, Randall, Brocki y Kerns (2009) compararon 31 niños con TDAH-C y 31 niños de control, entre los 7 y 12 años de edad. Algo similar intentaron Dreschler, Rizzo y Steinhausen (2010), entre 34 niños con TDAH-C y 34 controles.

Un segundo trabajo de Child Neuropsychology lo publican más recientemente Goth-Owens, Martinez-Torteya, Martel y Nigg (2010), dedicado al subtipo inatento del TDAH en una muestra de 571 niños y adolescentes de la comunidad. El subtipo inatento se definió como una condición heterogénea en la que teniendo una significativa proporción de TDAH no se apreciaban síntomas hiperacti- 
vos ni impulsivos. Este subgrupo, concluyen Goth-Owens et al. (2010), puede representar una condición de niños inatentos distinta, caracterizada ésta por un pobre control cognitivo de la interferencia.

En nuestros datos, ambos subtipos presentan diferente perfil neuropsicológico, obtenido éste con la batería Luria-DNI (1991), así como también diferente perfil cognitivo en los subtests del WISC-R. Se trata de una muestra clínica, de entre 7 y 10 años de edad, que en la mayoría de los casos fueron remitidos a evaluación neuropsicológica desde el colegio por escaso rendimiento académico. Fueron clasificados en cuatro grupos mediante análisis de conglomerados, a partir de las puntuaciones obtenidas en las escalas ECI de profesores (Manga, González y Fournier, 2008).

De una muestra de 118 niños, 50 fueron clasificados como TDAH-C y 20 como TDAH-I. La diferencia de perfiles neuropsicológicos entre el subtipo TDAH-C y el TDAH-I puede concretarse en que ambos subtipos coinciden en puntuar especialmente bajo en Regulación verbal de acto motor y en Escritura, pero son diferentes en Capacidad lingüística y Memoria.

La diferencia entre los dos subtipos se halla en la Memoria inmediata, superior ésta en el subtipo TDAH-I. Lo mismo se puede decir del subtest Denominación y habla narrativa. "Hay un acercamiento que tiende a relacionar los problemas de memoria de los niños con dificultades de aprendizaje más con un déficit lingüistico subyacente que con un déficit atencional" (Manga et al., 2008, p. 556).

En capacidades cognitivas, el perfil de los subtests del WISC-R presentan un patrón ACID claro (puntuaciones inferiores en Aritmética, Claves, Información y Dígitos) sólo en el grupo con TDAH-I. El patrón ACID se suele encontrar en grupos con dificultades de aprendizaje (Manga y Fournier, 1997), que pueden asociarse con otros problemas tales como déficit de atención (Manga et al., 2008).

También nuestros resultados apuntan, como los de Goth-Owens et al. (2010), a que los niños inatentos sin hiperactividad/impulsividad pueden caracterizarse por un pobre control cognitivo de la interferencia, a cuyo déficit se asocia el patrón ACID del WISC-R y dificultades de aprendizaje. No obstante, algún autor (v.g., Furman, 2008) está en contra de la existencia del TDAH como trastorno identificable, ya que la inatención, la hiperactividad y la impulsividad son síntomas de muchas condiciones médicas, emocionales y psicosociales que afectan a los niños.

\section{La memoria verbal inmediata}

Efectos de posición serial en la memoria de recuerdo libre. Hablamos de la denominada curva de posición serial (CPS). Se pueden estudiar los efectos de posición serial, la primacía y la recencia, en investigaciones neuropsicológicas (Franzen, 1989) sobre memoria inmediata.

El recuerdo libre es tema de estudio actualmente en los escolares de 8 a 10 
años (Lehmann y Hasselhorn, 2010), en cuyo campo interesan los cambios en el uso de estrategias de memoria durante este periodo del desarrollo. Son estrategias cada vez más sofisticadas a medida que los niños van creciendo. El repaso de elementos a memorizar y su influencia en la retención o mantenimiento de los mismos, en tareas verbales de recuerdo libre, tienen especial importancia cuando se relacionan con la capacidad de memoria a corto plazo de los individuos, en particular en estas etapas del desarrollo. Los resultados de Sasaki (2009) sugieren que los individuos con recencia poderosa tendrán también mayor capacidad en su memoria de trabajo (MT).

\section{LLAMADAS RECIENTES A FAVOR DE LA NEUROPSICOLOGÍA ESCOLAR}

La neuropsicología escolar, 30 años después de sus inicios, cuenta ya desde 2005 con un manual que recoge toda una serie de trabajos útiles para el profesional de la psicología que elija formarse adecuadamente en ella (véase, D’Amato, Fletcher-Janzen y Reynolds, 2005). Desde la década de 1980 se recomendaba a los psicólogos escolares una preparación adecuada en neuropsicología. Actualmente surgen invitaciones a la práctica de la neuropsicología infantil en las escuelas.

Murphy y Benton (2010), por ejemplo, se refieren a la nueva frontera de la neuropsicología educativa, en un número especial dedicado al tema por la revista Contemporary Educational Psychology. La neuropsicología es, según dicen, una fuente potencial de conocimiento científico y posee métodos capaces de ayudar a los psicólogos de la educación a conseguir una comprensión más profunda y significativa de la cognición, la motivación y el aprendizaje de los estudiantes. La fusión de la educación con la neuropsicología representa la más nueva frontera para los psicólogos de la educación.

Cleary y Scott (2011) acaban de hacer un llamamiento a favor de la implantación efectiva de la neuropsicología escolar. Se les dice a los psicólogos escolares que aprovechen las aportaciones de la neuropsicología clínica infantil.

\section{A MODO DE CONCLUSIÓN: LOS AVANCES EN LAS TÉCNICAS DE NEUROIMAGEN}

Como nos recuerdan recientemente quienes han revisado la neuropsicología aplicada al trabajo con niños (Witsken et al., 2008), hay que resaltar que han sido los avances en las técnicas de neuroimagen las que han permitido comprobar cómo cambia el cerebro a causa de las intervenciones neuropsicológicas. Las imágenes del funcionamiento cerebral muestran claramente los cambios surgidos como consecuencia del tratamiento neuropsicológico, ya que se consigue una reorganización del cerebro. Desde la universidad de París, Billard y Jambaque (2008) resaltan los rápidos avances de la neuropsicología infantil en los últimos años y la importancia de los procesos de maduración y plasticidad cerebrales. 
Son necesarios puentes de unión de los psicólogos de la educación con la neuropsicología infantil, para mejor conocer los cambios que ocurren en la organización funcional del cerebro en desarrollo (Casey, Galvan y Hare, 2005). El córtex prefrontal requiere un desarrollo prolongado, desarrollo que juega un importante papel en la maduración de las capacidades cognitivas superiores.

\section{REFERENCIAS}

Anderson, P. (2002). Assessment and development of executive function (EF) during childhood. Child Neuopsychology, 8 (2), 71-82.

Bausela, E. (2008). Estudio de validación de la batería Luria-Inicial en una muestra de escolares mexicanos. Revista Mexicana de Neurociencia, 9 (2), 125 130 .

Billard, C. y Jambaque, I. (2008). The rapid development of child neuropsychology. Revue Neurologique, 164, S108-S113.

Casey, B. J., Galvan, A. y Hare, T. A. (2005). Changes in cerebral functional organization during cognitive development. Current Opinion in Neurobiology, 15, 239-244.

Chevalier, N. (2010). Executive functions in children: concepts and developments. Canadian Psychology - Psychologie Canadienne, 51 (3), 149-163.

Christensen, A. L. (1975). Luria's neuropsychological investigation. Munksgaard, Copenhagen, Denmark.

Christensen, A.L. (1987). El diagnóstico neuropsicológico de Luria. Madrid: Visor. (2. ${ }^{\text {a }}$ ed. revisada).

Cleary, M. J. y Scott, A. J. (2011). Developments in clinical neuropsychology: Implications for school psychological services. Journal of School Health, 81 (1), 1-7.

D’Amato, R. C., Crepeau-Hobson, F., Huang, L. V. y Geil, M. (2005). Ecological neuropsychology: An alternative to de deficit model for conceptualizing and serving students with learning disabilities. Neuropsychology Review, 15 (2), 97-103.

D’Amato, R. C., Fletcher-Janzen, E. y Reynolds, C. (Eds.) (2005). Handbook of school neuropsychology. Nueva York: Wiley.

Das, J. P. (1999). A Neo-Lurian approach to assessment and remediation. Neuropsychology Review, 9 (2), 107-116.

Das, J. P., Naglieri, J. A., y Kirby, J. R. (1994). Assessment of cognitive processes: The PASS theory of intelligence. Boston: Allyn and Bacon.

Das, J. P., y Varnhagen, C. K. (1986). Neuropsychological functioning and cognitive processing. En J. E. Obrzut y G. W. Hynd (Eds.), Child neuropsychology, Vol. 1 (pp. 117-140). Orlando: Academic Press.

Dreschler, R., Rizzo, P. y Steinhausen, H. C. (2010). The impact of the instruction and response cost on the modulation of response-style in children with ADHD. Behavioral and Brain Functions, 6, $\mathrm{n}^{\circ} 31$.

Franzen, M.D. (1989). Reliability and validity in neuropsychological assessment. 
Nueva York: Plenum.

Furman, L. M. (2008). Attention-deficit hyperactivity disorder: Does new research support old concepts? Journal of Child Neurology, 23 (7), 775-784.

Gaddes, W. H. (1980). Learning disabilities and brain function: A neuropsychological approach. Nueva York: Springer-Verlag.

Gaddes, W. H. (1981). An examination of the validity of neuropsychological knowledge in educational diagnosis and remediation. En G. W. Hynd y J. E. Obrzut (Eds.), Neuropsychological assessment and the school-age child (pp. 27-84). Nueva York: Grune and Stratton.

Glozman, J. M. (1999). Quantitative and qualitative integration of Lurian procedures. Neuropsychology Review, 9 (1), 23-32.

Golden, C. J. (1981). The Luria-Nebraska Children's Battery: Theory and formulation. En G. W. Hynd y J. E. Obrzut (Eds.), Neuropsychological assessment and the school-age child (pp. 277-302). Nueva York: Grune and Stratton.

Goldstein, S. y Naglieri, J. (2008). The school neuropsychology of ADHD: Theory, assessment and intervention. Psychology in the Schools, 45 (9), 859-874.

Goth-Owens, T. L., Martinez-Torteya, C., Martel, M. M. y Nigg, J. T. (2010). Processing weakness in children and adolescents with non-hyperactive but inattentive ADHD (ADD). Child Neuopsychology, 16 (6), 577-591.

Hynd, G. W. y Obrzut, J. E. (1981). School neuropsychology. Journal of School Psychology, 19, 45-50.

Hynd, G. W. y Willis, W. G. (1988). Pediatric neuropsychology. Nueva York: Grune \& Straton.

Jurado, M. B. y Roselli, M. (2007). The elusive nature of executive functions: A review of our current understanding. Neuropsychology Review, 17, 213-233.

Kaufman, A.S. y Kaufman, N.L. (1983). K-ABC. Kaufman assessment battery for children. Interpretative manual. Cicle Pines: American Guidance Service.

Korkman, M. (1988). NEPSY: An adaptation of Luria's investigation for young children. The Clinical Neuropsychologist, 2, 375-392.

Korkman, M. (1999). Applying Luria's diagnostic principles in the neuropsychological assessment of children. Neuropsychology Review, 9 (2), 89-105.

Korkman, M., Kirk, U. y Kemp, S. L. (1998). NEPSY. A developmental neuropsychological assessment. The psychological Corporation, San Antonio, TX.

Lehmann, M. y Hasselhorn, M. (2010). The dynamics of free recall and the relation to rehearsal between 8 and 10 years of age. Child Development, 81 (3), 1006-1020.

Luria, A. R. (1973). The working brain: An introduction to neuropsychology. Nueva York: Basic Books. (Versión castellana: El cerebro en acción. Barcelona. Fontanella, 1979).

Luria, A. R. (1980). Higher cortical functions in man (2 $2^{\text {nd }}$ ed.). Nueva York: Basic Books.

Manga, D. y Fournier, C. (1997). Neuropsicología clínica infantil. Estudio de casos en edad escolar. Madrid: Universitas.

Manga, D. y Ramos, F. (1991). Neuropsicología de la edad escolar. Aplicaciones 
de la teoría de A. R. Luria a niños a través de la batería Luria-DNI. Madrid: Visor.

Manga, D. y Ramos, F. (1999). Evaluación neuropsicológica. Clínica y Salud, 10, 331-376.

Manga, D. y Ramos, F. (2000). Luria-DNA. Diagnóstico neuropsicológico de adultos. Madrid: TEA Ediciones.

Manga, D. y Ramos, F. (2001). Evaluación de los síndromes neuropsicológicos infantiles. Revista de Neurología, 32, 664-675.

Manga, D. y Ramos, F. (2006). Luria Inicial. Evaluación neuropsicológica en la edad preescolar. Madrid: TEA Ediciones.

Manga, D., González, H. y Fournier, C. (2008). Trastornos por déficit de atención en la infancia. En A. Belloch, B. Sandín y F. Ramos (Eds.), Manual de psicopatología, vol. 2 (pp. 549-566). Madrid: McGraw Hill.

Murphy, P. K. y Benton, S. L. (2010). The new frontier of educational neuropsychology: unknown opportunities and unfulfilled hopes. Contemporary Educational Psychology, 35, 153-155.

Obrzut, J. E. (1981). Neuropsychological procedures with school-age children. En G. W. Hynd y J. E. Obrzut (Eds.), Neuropsychological assessment and the school-age child (pp. 237-275). Nueva York: Grune and Stratton.

Obrzut, J. E. y Obrzut, A. (1982). Neuropsychological perspectives in pupil services: Practical application of Luria's model. Journal of Research and Development in Education, 15, 38-47.

Quintanar, L., Solovieva, Y., Lázaro, E., Bonilla, M. R., Mejía, L. y Eslava, J. (2008). Dificultades en el proceso lectoescritor. Laguna de Duero (Valladolid): Editorial de la Infancia

Ramos, F. y Manga, D. (2003). Evaluación neuropsicológica en niños: La Batería Luria-DNI y sus aplicaciones. En Fundación Mapfre Medicina, Neuropsicología Infantil (pp. 71-99). Madrid: MAPFRE.

Randall, R. D., Brocki, K. C. y Kerns, K. A. (2009). Cognitive control in children with ADHD-C: How efficient are they? Child Neuopsychology, 15 (2), 163-178.

Sasaki, T. (2009). Individual differences in working memory capacity in recency effects from the recall process. Psychological Reports, 104 (2), 545-548.

Semrud-Clikeman, M. y Teeter, P. A. (2009). Child neuropsychology. Assessment and interventions for neurodevelopmental disorders ( $2^{\text {nd }}$ ed.). Nueva York: Springer.

Shue, K. L. y Douglas, V. I. (1992). Attention deficit hyperactivity disorder and the frontal lobe syndrome. Brain and Cognition, 20, 104-124.

Stuss, D. T. y Benson, D. F. (1990). The frontal lobes and language. En E. Goldberg (Ed.), Contemporary neuropsychology and the legacy of Luria (pp. 29-50). Hillsdale, NJ : Erlbaum.

Tranel, D. (2005). On the use of neuropsychology to diagnose brain damage and study brain-behavior relationships: A comment on Luria. Cortex, 41, 259262 . 
Tupper, D. E. (1999). Introduction: Neuropsychological assessment après Luria. Neuropsychology Review, 9 (2), 57-61.

Witsken, D., Stoeckel, A. y D'Amato, R. C. (2008). Leading educational change using a neuropsychological response-to-intervention approach: linking our past, present, and future. Psychology in the Schools, 45(9), 781-798. 